\title{
Trastuzumab in combination with metronomic cyclophosphamide and methotrexate in patients with HER-2 positive metastatic breast cancer
}

\author{
Laura Orlando*1, Anna Cardillo1, Raffaella Ghisini1 ${ }^{1}$, Andrea Rocca1, \\ Alessandra Balduzzi ${ }^{1}$, Rosalba Torrisi ${ }^{1}$, Giulia Peruzzotti ${ }^{1}$, Aron Goldhirsch ${ }^{2}$, \\ Elisabetta Pietri ${ }^{1}$ and Marco Colleoni ${ }^{1}$
}

\begin{abstract}
Address: ${ }^{1}$ Unit of Research in Medical Senology, Department of Medicine, European Institute of Oncology, Via Ripamonti 435, 20141 Milan, Italy and 2Division of Medical Oncology, Department of Medicine, European Institute of Oncology, Via Ripamonti 435, 20141 Milan, Italy

Email: Laura Orlando* - laura.orlando@ieo.it; Anna Cardillo - anna.cardillo@ieo.it; Raffaella Ghisini - raffaella.ghisini@ieo.it; Andrea Rocca - andrea.rocca@ieo.it; Alessandra Balduzzi - alessandra.balduzzi@ieo.it; Rosalba Torrisi - rosalba.torrisi@ieo.it; Giulia Peruzzotti - giulia.peruzzotti@ieo.it; Aron Goldhirsch - aron.goldhirsch@ibcsg.org; Elisabetta Pietri - elisabetta.pietri@ieo.it; Marco Colleoni - marco.colleoni@ieo.it

* Corresponding author
\end{abstract}

Published: 15 September 2006

BMC Cancer 2006, 6:225 doi:10.1186/147I-2407-6-225

This article is available from: http://www.biomedcentral.com/147I-2407/6/225

(c) 2006 Orlando et al; licensee BioMed Central Ltd.

This is an Open Access article distributed under the terms of the Creative Commons Attribution License (http://creativecommons.org/licenses/by/2.0), which permits unrestricted use, distribution, and reproduction in any medium, provided the original work is properly cited.
Received: 12 June 2006

Accepted: 15 September 2006

\begin{abstract}
Background: HER2/neu overexpression is linked to promotion of angiogenesis in breast cancer. We therefore tested the activity of the combination of Trastuzumab with metronomic, low dose chemotherapy with cyclophosphamide (CTX) and methotrexate (MTX) in metastatic breast cancer (MBC).

Methods: Between April 2002 and June 2005, twenty-two patients with metastatic breast cancer with the presence of overexpression or amplification of HER2-/neu, all pre-treated with trastuzumab plus other cytotoxics, were treated with trastuzumab $(6 \mathrm{mg} / \mathrm{kg}$ every three weeks) in combination with metronomic chemotherapy (MTX $2.5 \mathrm{mg}$, bid on Day I and Day 4 every week) and CTX (50 mg daily) (CM).

Results: The 22 enrolled patients are evaluable: most had an ECOG performance status of 0 (I7 pts), and all were pre-treated with chemotherapy for metastatic disease; 14 had progressive disease at study entry, and II had progressive disease during the last trastuzumab therapy. Metastatic sites included: lung (5 pts), liver (I 4 pts), bone (12 pts), lymph nodes (8 pts), central nervous system (CNS) ( 9 pts). We observed 4 partial remission (PR) (I8\%, 95\% Cl 5-40\%), 10 stable disease (SD) (46\%, 95\% Cl 24-68\%), and 8 PD (36\%, $\mathrm{Cl} 17-59 \%)$. The clinical benefit (RP plus RC plus SD for $\geq 24$ weeks) in all pts and in pts with disease resistant to previous trastuzumab therapy were $46 \%(95 \% \mathrm{Cl}, 24-68 \%)$ and $27 \%(95 \% \mathrm{Cl}, 6-61 \%)$, respectively. Median time to progression was 6 months and median duration of treatment was 5 months (range, 0,7 to 18.4 months and range, I to 18 months, respectively). Overall clinical toxicity was generally mild. Grade $\geq 2$ reversible liver toxicity and leukopenia were reported in 5 and 3 pts, respectively.
\end{abstract}

Conclusion: The combination of trastuzumab and metronomic chemotherapy is effective and minimally toxic in advanced breast cancer patients. The efficacy observed in patients with disease resistant to trastuzumab supports the need of larger trial to confirm a role of this combination to delay acquired trastuzumab resistance. 


\section{Background}

Malignant tumors secrete factors that enable them to trigger their own angiogenesis. The initiation of angiogenesis requires acquisition of the angiogenic phenotype through a series of molecular events leading to increased expression of angiogenic factors and down-regulation of natural inhibitors [1].

Her2/neu is a 185-kilodalton transmembrane receptor tyrosine kinase that belongs to the epidermal growth factor receptor family [2,3]. Tumor overexpression of HER2/ neu is present in about $30 \%$ of patients with breast cancer and is associated with a worse histological grade, decreased overall survival and altered sensitivity to chemotherapeutic agents $[4,5]$. Recently, Her2/neu has been implicated in tumor angiogenesis. Experimental studies suggest that neutralizing antibodies against Her2/neu or EGFR results in down-regulation of angiogenesis, through VEGF gene suppression [6]. It is reported in the literature that such interaction occurs via abrogation of the increased synthesis of HIF1a (hypoxia inducible factor$1 a$ ) induced by c-erbB2 activation by ligands (i.r. heregulin) [7]. Moreover, a hypoxic-independent mechanims has been recently advocated in the angiogenetic involvement of HER2-/neu [8].

Trastuzumab (Herceptin ${ }^{\circledast}$; Genentech, South San Francisco, CA), a recombinant humanized anti-erbB2/HER-2 monoclonal antibody (MoAb) used in erbB2-overexpressing breast carcinoma, has been shown to have antiangiogenic properties [9]. Trastuzumab can induce normalization and regression of the vasculature in an experimental human breast tumor which overexpresses HER2 in mice, by modulating the effects of different proand anti-angiogenic factors [9]. The combination of trastuzumab with chemotherapeutic agents (paclitaxel, docetaxel) has been shown to increase the efficacy of trastuzumab in reducing angiogenesis in erbB2-overexpressing cells more than either therapy alone both in animal models and clinical studies [10,11].

We previously demonstrated the antitumor activity of oral low-dose methotrexate and cyclophosphamide delivered as metronomic chemotherapy in metastatic breast cancer and we have shown the correlation with vascular endothelial growth factor levels $[12,13]$. Other authors previously showed that long-term, low dose chemotherapy could elicit an antiangiogenic effect [14].

Based on these considerations, we evaluated the activity and tolerability of the combination trastuzumab plus low-dose oral cyclophosphamide and methotrexate in patients with metastatic breast cancer with overexpression or amplification of Her2-/neu and pre-treated with trastuzumab.

\section{Methods \\ Patient selection}

Patients included were required to have histologically confirmed metastatic breast carcinoma that either had, or had not, progressed after a line of trastuzumab alone or in combination with chemotherapy for metastatic disease. Other inclusion criteria were: measurable disease, age $\leq 80$ years, performance status ECOG $<3$, adequate bone marrow reserve defined as white blood cells $>4,000 \mathrm{~mm}^{3}$ and platelets $>100,000 \mathrm{~mm}^{3}$, adequate renal function (serum creatinine $<120 \mu \mathrm{mol} / \mathrm{l}$ ) and hepatic function (serum bilirubin $<20 \mu \mathrm{mol} / \mathrm{l}$, AST (SGOT) < $60 \mathrm{IU} / \mathrm{l})$. It was mandatory that all patients had to have recovered from any prior chemotherapy, radiotherapy, or surgery before the start of treatment.

Each patient included in this study gave their written informed consent. This protocol was notified to Ethical Committee.

\section{Evaluation and treatment}

Baseline evaluation included clinical examination, chest $\mathrm{X}$-ray, liver ultrasound or CT scan, bone nuclear scan (plus segmental bone radiographs when bone scans were positive), ECG, echocardiography with LVEF evaluation, and complete biochemical and hematological tests. A complete blood count was repeated every 14 days and biochemical tests every 28 days. Cardiac assessment via echocardiography with LVEF evaluation was performed every 3 months.

HER2/neu overexpression was assessed on routinely processed, formalin-fixed, paraffin-embedded tissue by immunohistochemical investigations using an automated immunostainer (TechMate 500; Dako, Glostrup, Denmark) and a peroxidase-based detection system in kit form (ChemMate; Dako) according to the manufacturer's instructions. The primary specific monoclonal antibody used was clone CB11; Biogenex. The FISH test was performed on routinely processed, formalin-fixed, paraffinembedded tissues using PathVision Her-2 DNA Probe Kit (Vysis Inc, Doweners Grove I11, and Inform HER-2 Gene Detection System, Ventana Medical Systems, Inc, Tucson, Arizona)

Patients were treated with MTX orally at a dose of $2.5 \mathrm{mg}$ twice a day on day 1 and 4 every week (10 am, 5 pm) and CTX orally at a dose of $50 \mathrm{mg}$ a day ( $9 \mathrm{am}$ ) in combination with Trastuzumab (Herceptin ${ }^{\circledast} 6 \mathrm{mg} / \mathrm{kg}$ every three weeks, after a loading dose of $8 \mathrm{mg} / \mathrm{kg}$ at the first administration)

\section{Side effects and response}

Toxicity was evaluated according to National Cancer Institute's (NCI) Common Terminology Criteria for Adverse Events (CTCAE) version 2.0 
Treatment was withheld and delayed for 1 week in cases of a neutrophil count of less than 1,000 $\mathrm{mm}^{3}$ and/or platelet count less than $75,000 \mathrm{~mm}^{3}$. A $50 \%$ dose reduction in the total amount of drug administered in each cycle was prescribed after hematological recovery. In cases of a neutrophil count less than 1,500 $\mathrm{mm}^{3}$ but $>1,000$ $\mathrm{mm}^{3}$ and/or platelet count less than $100,000 \mathrm{~mm}$ but $>75,000 \mathrm{~mm}^{3}$ therapy was administered with a 50\% dose reduction in the total amount of drug administered in each cycle. Re-escalation of drug doses was attempted if close monitoring was possible.

In the event of grade $\geq 2$ anorexia, nausea, vomiting, diarrhea, stomatitis, dryness of the mouth, epigastric pain, increase in transaminases, all therapy was postponed until symptoms subsided. A 50\% reduction of CM was performed for the next cycle, with subsequent re-escalation to full dosage if tolerated. Any other non-hematological Grade 3 toxicity was managed by a $50 \%$ reduction of dosage in the next cycle, which was not commenced until full recovery had occurred. Cardiac function was monitored with echocardiography every 3 months and therapy with Herceptin was interrupted for values of Ejection Fraction (EF) below 50 or in cases of reduction of $\leq 10 \%$ from baseline value.

Assessment of response was conducted according to WHO criteria and was performed after every 8 weeks of therapy. CR was defined as the disappearance of all known lesions on two separate measurements at least 4 weeks apart. PR was defined as a reduction of each lesion by at least $50 \%$. SD was defined as a decrease of less than $50 \%$ or an increase of less than $25 \%$ with no new lesions, and progressive disease as an increase of more than $25 \%$ or appearance of new lesions. Clinical benefit was defined as the proportion of patients who achieved CRs, PRs, or SDs for at least 24 weeks.

\section{Statistical analysis}

The study was conducted with a two-stage design according to Gehan. In order to attain a power of 0.9 to detect a response rate of 20\%, 11 patients are required in the first stage and if none demonstrates a response the study must be closed because of lack of activity. Because two patients responded, a further eleven patients were required to reach a precision of 0.1 in the estimate of the true response rate. Confidence intervals for the response rates were calculated using exact binomial methods.

Estimated curves of time to progression were plotted from the first day of treatment by the method of Kaplan and Meier; response duration was measured from the date of achievement of response. The association between categorical variables was assessed by the Fisher exact test.

\section{Results}

Between April 2002 and June 2005, twenty two metastatic breast cancer patients were treated with the combination of trastuzumab and metronomic chemotherapy. All patients but one demonstrated overexpression of Her2-/ neu $(3+)$ (seven patients determined in the primary $(32 \%)$ and fifteen patients in the metastatic sites (68\%). The FISH test was performed in the tumor with overexpression of Her2/neu (2+) and resulted positive for amplification of the gene. Progressive disease at study entry was registered in 14 patients; among these, 11 patients had progressive disease during trastuzumab-containing regimens as the last treatment before study entry. Moreover, one patient had progressive disease during the last trastuzumab-containing therapy (not the last treatment before study entry) (Table 1). Previous trastuzumab-containing regimens are showed in Table 1. Median follow-up was 15 months. All patients had measurable disease.

Oral cyclophosphamide plus methotrexate in combination with trastuzumab produced 4 PRs providing an objective response rate of $18 \%$ (95\% CI 5-40\%). An additional 10 patients had stabilization of disease and of these, 6 patients showed long-term disease stabilization (SD after 24 weeks), providing an overall clinical benefit (CR + PR + SD after 24 weeks) of 46\% (95\% CI 24-68\%). Previous treatments in patients achieving clinical benefit are summarized in Table 2.

Patients achieving clinical benefit were all pre-treated with $\geq 2$ lines of chemotherapy for metastatic disease and with at least one line of Trastuzumab containing regimen. The clinical benefit in patients with progressive disease at study entry was 36\% (95\% CI,13-65\%) (Table 2). Two patients among those who achieved PR and three patients among those who achieved prolonged disease stabilization had progressive disease at study entry. In particular, 3 patients had progressive disease to trastuzumab-containing combinations and 2 to trastuzumab-non containing regimen. Moreover one patient had progressive disease to the last trastuzumab-containing therapy (not performed as the last therapy before study entry). The clinical benefit in patients who progressed during the trastuzumab-containing regimen (trastuzumab-resistant) was 27\% (3 pts out of $11,95 \%$ CI, 6-61\%).

Exploratory analysis on features associated with the achievement of a clinical benefit showed a trend toward a larger percentage of clinical benefit among patients who received only one previous line of trastuzumab plus chemotherapy $(62 \%, 8$ out of 13$)$ compared with those who received more than one previous line $(22 \%, 2$ out of 9) 
Table I: Patients characteristics

\begin{tabular}{|c|c|c|}
\hline N. Patients & & 22 \\
\hline Median age, years (range) & & $48.9(31-70)$ \\
\hline \multicolumn{3}{|l|}{ Progressive disease at study entry } \\
\hline & No & 8 \\
\hline & Yes & 14 \\
\hline Progressive disease to Her combination & & 7 \\
\hline Progressive disease to Her alone & & 4 \\
\hline Progressive disease to non Her containing therapy* & & 3 \\
\hline \multicolumn{3}{|l|}{ Number of sites } \\
\hline & I & 1 \\
\hline & 2 & 9 \\
\hline & $\geq 3$ & 12 \\
\hline \multicolumn{3}{|l|}{ Tumor sites } \\
\hline & Lung & 5 \\
\hline & Liver & 14 \\
\hline & Bone & 12 \\
\hline & Lymphnodes & 8 \\
\hline & CNS & 9 \\
\hline & Other & 10 \\
\hline Previous treatment with Herceptin alone & & 1 \\
\hline \multirow[t]{4}{*}{ Previous treatment with Herceptin in combination } & & 16 \\
\hline & I line & 8 \\
\hline & 2 lines & 4 \\
\hline & 3 lines & 4 \\
\hline \multirow[t]{3}{*}{ Previous treatment with Herceptin alone and Herceptin in combination } & & 5 \\
\hline & 2 lines & 4 \\
\hline & 3 lines & 1 \\
\hline Herceptin + Vinorelbine & & 11 \\
\hline Herceptin + Taxanes & & 15 \\
\hline Herceptin + Others & & 5 \\
\hline ER and PgR negative & & 15 \\
\hline ER and/or PgR positive & & 7 \\
\hline \multicolumn{3}{|l|}{ Ki 67} \\
\hline & $<20 \%$ & 1 \\
\hline & $\geq 20 \%$ & 12 \\
\hline & Unknown & 9 \\
\hline \multicolumn{3}{|l|}{ Her2/Neu overexpression } \\
\hline & $2+$ & 1 \\
\hline & $3+$ & 21 \\
\hline
\end{tabular}

*one also progressed to the last trastuzumab-containing regimen

Median time to response was 2 months and, among responding patients, the median duration of response was 8.8 (range: $1-12+$ months.). Median time to progression was 6 months (range $0.7-18.4+; 95 \% \mathrm{CI}, 3.6$ to inf) and median duration of treatment was 5 months (range, 1 to $18+$ months).

A total of 149 months of therapy were administered, median per patient 5 months. Only 3 patients $(13.6 \%)$ had delayed chemotherapy and only 3 (13.6\%) reduced dosages of MTX. Reasons for delay and dose reduction was mainly leukopenia and increase in transaminases. Table 3 summarizes the side effects observed. Treatment was well tolerated. The most frequently encountered toxicity was grade II leukopenia, which was observed in $14 \%$ of the cases. Increase in transaminase values were registered in 8 cases $(36 \%)$ ( 2 cases with grade III).

No cardiotoxicity was reported with the exception of one patient with decreasing value of ejection fraction (EF) to $51 \%$ (less than $10 \%$ from baseline value) after one month of the trastuzumab therapy plus CM and after more than 1 year of the other trastuzumab-chemotherapy combinations.

\section{Discussion}

The combination of trastuzumab with chemotherapeutic agents is a well established approach for treatment of HER2 positive metastatic breast cancer. Preclinical models and subsequent clinical data have demonstrated an addi- 
Table 2: Previous treatments in patients achieving clinical benefit

\begin{tabular}{cccc}
\hline Best response & $\begin{array}{c}\text { Progression at study } \\
\text { entry }\end{array}$ & $\begin{array}{c}\text { Previous lines of chemotherapy for metastatic disease } \\
\text { Previous Trastuzumab-lines for metastatic disease }\end{array}$ \\
\hline$S D \geq 24$ weeks & NO & 3 & 3 \\
$S D \geq 24$ weeks & NO & 4 & 1 \\
$S D \geq 24$ weeks & YES* & 4 & 1 \\
$S D \geq 24$ weeks & NO & 3 & 1 \\
$S D \geq 24$ weeks & YES & 4 & 1 \\
SD $\geq 24$ weeks & YES** & 4 & 2 \\
PR & YES* & 3 & 2 \\
PR & NO & 8 & 3 \\
PR & NO & 2 & 1 \\
PR & YES* & 5 & 2 \\
\hline
\end{tabular}

* Progression at study entry to Trastuzumab-containing regimen

** Progression to the last Trastuzumab-containing regimen

tive or synergistic effect of the combination with platinum salts, paclitaxel, docetaxel, vinorelbine or more than one drugs [15-18].

Unfortunately, the success of these combinations in responding patients is compromised by the development of acquired resistance [4]. The mechanism of resistance to trastuzumab in animal models is the consequence of heritable genetic alterations and involved different, independent mechanisms [19]. The opportunity, in patients with progressive disease, of continuing trastuzumab combined with a non cross resistant chemotherapeutic regimen is a crucial question regarding trastuzumab strategy. Data on restored efficacy of trastuzumab with further associations after failure are limited, although some activity was recently reported in patients with progressive disease during trastuzumab therapy regarding a further non cross resistant chemotherapeutic regimen in combination with trastuzumab [20-22].

Recently, Kerbel et al reported increased angiogenic activity, as demonstrated by the up-regulation of VEGF, in tumors resistant to trastuzumab therapy in murine models [19]. Using this model, the authors showed that eventual relapses during trastuzumab therapy can be significantly delayed by prolonged combination therapy with metronomic low-dose cyclophosphamide chemotherapy. Another important issue is the preliminary evidence for the superiority of combining metronomic chemotherapy (cyclophosphamide) versus standard maximum tolerated dose (MTD) chemotherapy in terms of efficacy and toxicity.

The current trial, the only clinical experience reported of metronomic chemotherapy plus trastuzumab for patients with advanced breast cancer, indicates a role for this combination to possibly delay acquired resistance to trastuzumab, and to treat patients whose tumor variants have acquired resistance. In our series all patients had received trastuzumab before commencing the combination of metronomic chemotherapy and trastuzumab and 14 patients had progressive disease at their inclusion in the study. The majority of them were progressive during trastuzumab-containing regimen (11 patients) as the last therapy before study entry. We observed a clinically significant activity in both patients with progressive disease at study entry and in patients who had progressive disease during the last course of trastuzumab containing therapy. We have considered whether these results might be due to the effect of metronomic chemotherapy alone or due to a synergistic effect of the combination. In previous studies in which metronomic chemotherapy was used alone, we observed an overall response of $10 \%$ and a global clinical benefit of $32 \%$ in pre-treated patients $[12,13]$. In the present study, we observed an overall response of $18 \%$ and a clinical benefit (RP plus RC plus SD for $\geq 24$ weeks) of $46 \%$, confirming a significant activity of the combination of metronomic chemotherapy plus trastuzumab. Treatment beyond progression represents a new paradigm in cancer therapy, although the indirect comparison of the two studies (not obtained in a randomized setting) and the nature of the present study cannot justify the extended use of trastuzumab after progression or the use of the combination of trastuzumab with metronomic chemotherapy instead of the single modalities alone. Moreover, eight patients started the study protocol after achieving clinical response to previous trastuzumab combination therapy. The possibility that trastuzumab alone could have allowed a clinical benefit similar to that obtained with trastuzumab plus metronomic chemotherapy is reasonable.

Additional studies to test the independent contribution of metronomic chemotherapy and trastuzumab when used after disease progression during trastuzumab are warranted. 
Table 3: Side effects

\begin{tabular}{llll}
\hline & Grade I & Grade 2 & Grade 3 \\
\hline Leukopenia & $\mathrm{I}$ & 3 & - \\
Neutropenia & $\mathrm{I}$ & $\mathrm{I}$ & - \\
Nausea/Vomiting & 2 & - & - \\
Mucositis & $\mathrm{I}$ & - & - \\
Transaminases & 4 & 2 & 2 \\
Asthenia & 2 & $\mathrm{I}$ & - \\
Cardiac & 0 & 0 & 0 \\
\hline
\end{tabular}

Among patients with progressive disease at study entry we observed 2 objective responses and 3 cases of prolonged stabilization of disease indicating an activity in terms of clinical benefit in resistant patients ( 5 out of 14 patients, $36 \%$ ) (Table 2). Moreover, in 6 patients we registered no evidence of progressive disease for at least 24 weeks indicating that a small but relevant fraction of heavily pretreated MBC patients can achieve long term clinical benefit with this treatment option.

Among patients in whom progressive disease was recorded during the last course of trastuzumab containing therapy (11 pts) we observed 2 PR and 1 prolonged SD, for an overall clinical benefit of $27 \%$. These results are particularly relevant since they support, in a clinical setting, the combination of metronomic low-dose cyclophosphamide and methotrexate plus trastuzumab, as a possible strategy for delaying or treating acquired resistance to trastuzumab in human breast cancer xenografts, as previously published [19], although the nature of our study and the small sample size do not allow definitive conclusions on this issue.

The majority of patients (12 patients, $54.5 \%$ ) of our series were pre-treated with more than 2 lines of chemotherapy (in combination or not with trastuzumab) and among these, 5 patients had previously received 4 lines. The chance of obtaining a long term clinical benefit after more than 2 lines of chemotherapy in metastatic breast cancer is usually thought to be very poor, in particular for patients with HER2/neu positive MBC characterized by an aggressive disease course [23]. Moreover, in the present series, 9 patients had CNS involvement. Among these, 6 achieved clinical benefit. The inclusion of these patients, for whom a local treatment (radiotherapy in the majority of cases) was also planned or previously performed, was allowed due to the ability of both CTX and MTX to pass the BBB (blood-brain barrier) and to have activity in brain metastases from breast cancer [24], although there are not data of efficacy with the use of low dose metronomic chemotherapy.
The palliative goal of treatment in metastatic breast cancer and the achievement of symptomatic control and maintenance of quality of life are desirable treatment end points for the individual patient. Within this context the results achieved indicate that metronomic chemotherapy in combination with trastuzumab might represent a relevant additional therapeutic tool. In fact patients with HER2/ neu positive $\mathrm{MBC}$, where long term treatment with trastuzumab has been demonstrated to be feasible and safe [25], may benefit from combination with drugs characterized by a low incidence of toxic effects to allow the long term delivery of the combination.

As previously mentioned, in our series toxicity was mild. The profile of side effects was in keeping with previously published data on metronomic chemotherapy and included only $18 \%$ of grade $>2$ leucopenia or neutropenia, and only $27 \%$ of non hematologic toxicity. The addition of trastuzumab does not seem to increase the systemic toxicity and the individual tolerability. Only one patient, treated with trastuzumab for more than 1 year before the addition of $\mathrm{CM}$, experienced a mild decreased of ventricular left ejection fraction (less than 10\% from baseline value) and trastuzumab was interrupted.

\section{Conclusion}

Low-dose, oral cyclophosphamide and methotrexate combined with trastuzumab demonstrated substantial efficacy in metastatic breast cancer and provided control of the disease (clinical benefit) in a significant proportion of patients. Although derived from a small non randomized study, our results could provide further support for the disputable approach of treating breast cancer patients with her2/neu positive disease using trastuzumab- containing therapies beyond progression. Due to the theoretical background and the promising results reported in this study, the value of the combination of metronomic chemotherapy and trastuzumab should be further explored in larger clinical trials focused on the debatable attitude to continue trastuzumab beyond progression.

\section{Abbreviations \\ CTX: cyclophosphamide}

MTX: methotrexate

MBC: metastatic breast cancer

CM: cyclophosphamide + methotrexate

PR: partial remission

SD: stable disease 
CNS: central nervous system

BBB (blood-brain barrier)

\section{Competing interests}

The author(s) declare that they have no competing interests.

\section{Authors' contributions}

LO conceived of the study, participated in design and coordination and prepare the manuscript, AC participated in the design of the study and in the data collection, RG participated in the data management, AR participated in the design of the study and performed the statistical analysis, AB participated in the data collection, RT participated in the data collection, GP participated in the data management, AG participated in the study design, EP participated in the data collection, MC conceived of the study, participated in design and coordination and prepare the manuscript

\section{All authors read and approved the final manuscript.}

Institutional funding was used by each authors for study design and conduction, data collection, statistical analysis, manuscript preparation

\section{Acknowledgements}

We thank our clinical and laboratory colleagues who have contributed to our research.

We thank Mr. W. Russell for editorial support

\section{References}

I. Hanahan D, Folkman J: Patterns and emerging mechanism of the angiogenic switch during tumorigenesis. Cell 1996, 86:353-364.

2. Yamamoto T, Ikawa S, Akiyama T, Semba K, Nomura N, Miyajima N, Saito T, Toyoshima K: Similarity of protein encoded by the human c-erbB2 gene to epidermal growth factor receptor. Nature 1986, 3 1 9:230-234.

3. Bargmann $\mathrm{Cl}$, Hung $\mathrm{MC}$, Wienberg RA: The neu oncogene encodes an epidermal growth factor receptor-related protein. Nature 1986, 319:226-230.

4. Revillion F, Bonneterre J, Peyrat JP: ERBB2 oncogene in human breast cancer and its clinical significance. Eur J Cancer 1998, 34:79|-808.

5. Baselga J, Seidman AD, Rosen PP, Norton L: HER2 overexpression and paclitaxel sensitivity in breast cancer: therapeutic implications. Oncology 1997, I I:43-48.

6. Petit AM, Rak J, Hung MC, Rockwell P, Goldstein N, Fendly B, Kerbel RS: Neutralizing antibodies against epidermal growth factor and erbB-2/neu receptor tyrosine kinases down-regulate vascular endothelial growth factor production by tumor cells in vitro and in vivo. Angiogenic implications for signal transduction therapy of solid tumors. Am J Pathol 1997, | $51: 1523-1530$

7. Laughner E, Taghavi P, Chiles K, Mahon PC, Semenza GL: HER2 (neu) signalling increases the rate of hypoxia-inducible I alpha (HIF-Ialpha) synthesis:novel mechanism for HIF-Imediated vascular endothelial growth factor expression. Mol Cell Biol 200I, 2 I:3995-4004.

8. Yen L, Benlimane N, Nie ZR, Xiao D, Wang T, Al Moustafa AE, Esumi H, Milanini J, Hynes NE, Pages G, Alaoui-Jamali Ma: Differential reg- ulation of tumor angiogenesis by distinct erbB homo and heterodimers. Mol Biol Cell 2002, I 3:4029-4044.

9. Izumi Y, Xu L, di Tomaso E, Fukumura D, Jain RK: Herceptin acts as an anti-angiogenic cocktail. Nature 2002, 4 I 6:279-280.

10. Klos KS, Zhou X, Lee S, Zhang L, Yang W, Nagata Y, Yu D: Combined trastuzumab and paclitaxel treatment better inhibits erbB2 mediated angiogenesis in breast carcinoma through a more effective inhibition of AKT than either treatment alone. Cancer 2003, 98: | 377-I385.

II. Koukurakis MI, Simopoulos C, Polychronidis A, Perente S, Botaitis S, Giatromanolaki A, Sivridis E: The effect of trastuzumab/ docetaxel combination on breast cancer angiogenesis:dichotomus effect predictable by the HIFla/VEGF pretreatment status? Anticancer Res 2003, 23:1673-1680.

12. Colleoni M, Rocca A, Sandri MT, Zorzino L, Masci G, Nolè F, Peruzzotti G, Robertson C, Orlando L, Cinieri S, de Braud F, Viale G, Goldhirsch A: Low-dose oral methotrexate and cyclophosphamide in metastatic breast cancer:antitumor activity and correlation with vascular endothelial growth factor levels. Ann Oncol 2002, I3:73-80.

13. Colleoni M, Orlando L, Sanna G, Rocca A, Maissonneuve A, Sandri MTP, Peruzzotti G, Ghisini R, Sandri MT, Zorzino L, Nolè F, Viale G, Goldhirsch A: Metronomic low-dose oral cyclophosphamide and methotrexate plus or minus thalidomide in metastatic breast cancer: antitumor activity and biological effects. Annals Oncol 2006, I7(2):232-8.

14. Kerbel RS, Viloria-Petit A, Klement G, Rak J: "Accidental" antianiogenic drugs: anti-oncogene directed signal transduction inhibitors and conventional chemotharapeutic agents as examples. Eur J Cancer 2000, 36: I 248- 1257.

15. Slamon DJ, Leyland-Jones B, Shak S, Fuchs H, Paton V, Bajamonde A Fleming T, Eiermann W, Wolter J, Pegram M, Baselga J, Norton L: Use of chemotherapy plus a monoclonal antibody against HER2 for metastatic breast cancer that overexpresses HER2. N Engl J Med 200I, 344:783-792.

16. Burstein HJ, Kuter I, Campos SM, Gelman RS, Tribou L, Parker LM, Manola J, Younger J, Matulonis U, Bunnell CA, Partridge AH, Richardson PG, Clarke K, Shulman LN, Winer EP: Clinical activity of trastuzumab and vinorelbine in women with HER2 overexpressing metastatic breast cancer. J Clin Oncol 200I, 1 9:2722-2730.

17. Esteva FJ, Valero V, Bosser D, Guerra LT, Murray JL, Pusztai L, Cristofanilli M, Arun B, Esmaeli B, Fritsche HA, Sneige N, Smith TL, Hortobagyi GN: Phase II study of weekly docetaxel and trastuzumab for patients with HER2-overexpressing metastatic breast cancer. J Clin Oncol 2002, 20:1800-1808.

18. Pegram MD, Lipton A, Hayes DF, Weber BL, Baselga JM, Tripathy D, Baly D, Baughman SA, Twaddell T, Glaspy JA, Slamon DJ: Phase II study of receptor-enhanced chemosensitivity using recombinant humanized anti-p I 85 monoclonal antibody plus cisplatin in patients with HER2/neu overexpressing metastatic breast cancer refractory to chemotherapy treatment. J Clin Oncol 1998, 16:2659-267I.

19. du Manoir JM, Francia G, Man S, Mossoba M, Medin JA, Vigoria-Petit A, Hicklin DJ, Emmenegger U, Kerbel RS: Strategies for delaying or treating in vivo acquired resistance to trastuzumab in human breast cancer xenografts. Clin Cancer Res 2006, I 2(3):904-9|6.

20. Garcia-Saenz JA, Martin M, Puente J, Lopez-Tarruella S, Casado A, Moreno F, Grande E, Diaz-Rubio E: Trastuzumab associated with successive cytotoxic therapies beyond disease progression in metastatic breast cancer. Clin Breast cancer 2005, 6(4):325-329.

21. Gelmon KA, Mackey J, Verma S, Gertler SZ, Bangemann N, Klimo P, Schneeweiss A, Bremer K, Soulieres D, Tonkin K, Bell R, Heinrich B, Grenier D, Dias R: Use of trastuzumab beyond disease progression: observations from a retrospective review of case histories. Clin Breast Cancer 2004, 5:52-58.

22. Fountzilas G, Razis E, Tsavdaridis D, Karina M, Labropoulos S, Christodoulou C, Mavroudis D, Gogas H, Georgoulias V, Skarlos D: Continuation of trastuzumab beyond disease progression is feasible and safe in patients with metastatic breast cancer: a retrospective analysis of 80 cases by the Hellenic Cooperative Oncology Group. Clin Breast Cancer 2003, 4: I 20- I 25.

23. Slamon DJ, Clark GM, Wong SG, Levin WJ, Ullrich A, McGuire WL: Human breast cancer: correlation of relapse and survival 
with amplification of Her2/neu oncogene. Science 1987, 235: $177-182$.

24. Rosner $D$, Nemoto T, Lane WW: Chemotherapy induces regression of brain metastasis in breast carcinoma. Cancer 1986, 58(4):832-9.

25. Tripathy D, Slamon DJ, Cobleigh M, Arnold A, Saleh M, Mortimer JE, Murphy M, Stewart SJ: Safety of treatment of metastatic breast cancer with trastuzumab beyond disease progression. J Clin Oncol 2004, 22: 1063-1070.

\section{Pre-publication history}

The pre-publication history for this paper can be accessed here:

http://www.biomedcentral.com/1471-2407/6/225/pre pub

Publish with Bio Med Central and every scientist can read your work free of charge

"BioMed Central will be the most significant development for disseminating the results of biomedical research in our lifetime. " Sir Paul Nurse, Cancer Research UK

Your research papers will be:

- available free of charge to the entire biomedical community

- peer reviewed and published immediately upon acceptance

- cited in PubMed and archived on PubMed Central

- yours - you keep the copyright

Submit your manuscript here:

http://www.biomedcentral.com/info/publishing_adv.asp 